\title{
OPEN Physiological and molecular correlates of the screwworm fly attraction to wound and animal odors
}

\author{
Paul V. Hickner ${ }^{1}$, Omprakash Mittapalli $^{1}$, Anjana Subramoniam ${ }^{1}$, Agustin Sagel ${ }^{2}$, \\ Wes Watson ${ }^{3}$, Maxwell J. Scott $\mathbb{1}^{3}$, Alex P. Arp ${ }^{4}$, Adalberto A. Pérez de León ${ }^{4}$ \& \\ Zainulabeuddin Syed ${ }^{1} \otimes$
}

The screwworm fly, Cochliomyia hominivorax (Coquerel), was successfully eradicated from the United States by the sterile insect technique (SIT). However, recent detection of these flies in the Florida Keys, and increased risk of introductions to the other areas warrant novel tools for management of the flies. Surveillance, a key component of screwworm control programs, utilizes traps baited with rotting liver or a blend of synthetic chemicals such as swormlure-4. In this work, we evaluated the olfactory physiology of the screwworm fly and compared it with the non-obligate ectoparasitic secondary screwworm flies, $C$. macellaria, that invade necrotic wound and feed on dead tissue. These two species occur in geographically overlapping regions. C. macellaria, along with other blowflies such as the exotic $C$. megacephala, greatly outnumber $C$. hominivorax in the existing monitoring traps. Olfactory responses to swormlure-4 constituents between sex and mating status (mated vs unmated) in both species were recorded and compared. Overall, responses measured by the antennograms offered insights into the comparative olfactory physiology of the two fly species. We also present detailed analyses of the antennal transcriptome by RNA-Sequencing that reveal significant differences between male and female screwworm flies. The differential expression patterns were confirmed by quantitative PCR. Taken together, this integrated study provides insights into the physiological and molecular correlates of the screwworm's attraction to wounds, and identifies molecular targets that will aid in the development of odorant-based fly management strategies.

The larvae of the screwworm fly, Cochliomyia hominivorax (Coquerel, 1858), are obligate ectoparasites of warmblooded animals, with a historic range of subtropical and tropical regions of North and South America, and parts of the Caribbean. Great economic losses from screwworm to livestock production in the United States, estimated to be US\$ 70-100 million annually in the 1960s, inspired scientists with the U.S. Department of Agriculture (USDA) to develop the sterile insect technique (SIT) for the eradication of screwworm ${ }^{1,2}$. The C. hominivorax SIT program, considered as one of the most effective pest eradication programs ever conducted, has eradicated screwworm from all of North and Central America, as well as Curaçao, Puerto Rico, and an introduction to Libya $^{3}$. Presently, a barrier zone on the border of Panama and Colombia is maintained and co-managed by the USDA and the Panamanian Ministry of Agriculture (COPEG). The program consists of C. hominivorax massrearing facility, aerial dispersal center, and an extensive network of field personnel that respond to probable cases, conduct outreach and training with local ranchers, and operate livestock transportation check stations.

Effective and integrated pest management strategies depend on a strong understanding of the population dynamics. Blood feeding and parasitic insects display robust olfactory driven behaviors ${ }^{4,5}$, and exploiting the sense of smell (olfaction) is increasingly appreciated in developing the monitoring and management tools for forensic ${ }^{6}$ and medically important dipteran flies ${ }^{7}$. Routine testing of sterile C. hominivorax field performance and

\footnotetext{
${ }^{1}$ Department of Entomology, University of Kentucky, Lexington, KY 40546, USA. ${ }^{2}$ USDA, Agricultural Research Service, Knipling-Bushland U.S. Livestock Insects Research Laboratory and Veterinary Pest Genomics Center, Screwworm Research Site, Pacora, Panama. ${ }^{3}$ Department of Entomology and Plant Pathology, North Carolina State University, Campus Box 7613, Raleigh, NC 27695-7613, USA. ${ }^{4}$ USDA, Agricultural Research Service, Knipling-Bushland U.S. Livestock Insects Research Laboratory and Veterinary Pest Genomics Center, 2700 Fredericksburg Rd., Kerrville, TX 78028, USA. ${ }^{\circledR}$ email: zsy224@uky.edu
} 
capture of wild flies in outbreak or in non-eradicated areas utilize two trapping methods: vertical sticky traps baited with swormlure $-4^{8}$, or putrefied beef liver ${ }^{9}$. Development of these trapping systems evolved over the course of the screwworm eradication program spanning several decades. Early observation that $C$. hominivorax females were attracted to rotting liver ${ }^{10}$, animal wound $s^{11}$, bacterial contaminated bovine blood ${ }^{12}$, and fluid from sheep wounds infested with larva $\mathrm{e}^{13}$ led the way for isolating and identifying chemical constituents from these natural substrates. Chemical analyses of one such attractive oviposition substrate, incubated blood, identified phenol, 4-methylphenol ( $p$-cresol) and indole as major constituents ${ }^{14}$. Testing 35 compounds implicated as breakdown products of protein and fatty acids resulted in the development of a synthetic attractant blend, later called swormlure, that proved 5.7 times more effective to males and reduced the trapping of non-target species capture by $c a .87 \%$ in comparison with beef liver ${ }^{15}$. This blend was further improved by adding dimethyl disulfide and removing acetone, becoming swormlure- $2^{16,17}$. Due to environmental challenges as the screwworm eradication program pushed farther south into Mexico, swormlure-2 was not as effective. An adjustment to the release rates of the constituent compounds resulted in swormlure-4, the currently used attractant ${ }^{8}$.

A series of elegant experiments by Cork et al., starting with the chemical and electrophysiological analysis of the natural larval infested wound fluid (NWF) identified an array of electrophysiologically active compounds ${ }^{18}$. These compounds were subsequently evaluated and compared with the sentinel sheep, NWF and swomlure- $4^{19}$, and offered mixed results, especially in terms of sex ratios in trap captures. This study therefore highlighted the need for future research to account for the physiological responses from males and female of all reproductive status $^{19}$. Therefore, despite years of research into the chemical ecology of adult $C$. hominivorax towards developing a reliable and robust synthetic bait, routine surveillance of myiasis cases in the barrier zone and endemic areas still rely largely on liver bait stations and swormlure $-4^{3}$ as was recently demonstrated following the outbreak in Florida ${ }^{20}$. This points to the need for more comprehensive research strategies combining physiological and molecular analyses to identify robust and reliable baits.

In insects, physiological sensitivity and behavioral response to odors vary depending on the organism's physiological state such as feeding and nutritional status, mating status and sexual maturation ${ }^{21}$, as demonstrated in flies $^{22,23}$, moths ${ }^{24}$ and mosquitoes ${ }^{25}$. Screwworm flies are no exception. Male C. hominivorax do not need to feed on an animal protein source, thus male attraction to the compounds in swormlure is likely in search of females searching for an oviposition site or mimicking flowers that emit carrion odors ${ }^{26}$. In the females, responsiveness to odors such as those that indicate the nutritive or reproductive resources is modulated by oocyte maturation ${ }^{27}$. Additionally, different odor sources attracted females at different stages of ovarian development ${ }^{28}$. Whereas swormlure-4 is assumed to be equally attractive to nulliparous and parous $C$. hominivorax females, liver traps were most attractive to parous females likely looking for protein meals rather than oviposition sites ${ }^{15,16}$. In terms of sex differences in capture rates, swormlure-4 has been reported to attract an equal number of males and females $^{19,29}$, or biased towards females ${ }^{8}$.

We addressed these intriguing questions by employing a multipronged strategy. First, we measured the olfactory sensitivity of $C$. hominivorax males, virgin or gravid females to the constituent compounds of swormlure-4. Next, leveraging the newly sequenced genome assembly of this $\mathrm{fly}^{30}$, we isolated and analyzed the antennal transcriptome by RNA-Sequencing to gain insights into the molecular basis of olfaction in male and female screwworm flies. Antennal olfactory sensitivity is the outcome from the binding of odorants with the members of three divergent protein families-odorant receptors (ORs), ionotropic receptors (IRs), and gustatory receptors (GRs) - together referred as chemosensory gene families ${ }^{31}$. We previously identified 79 ORs, 84 GRs, 88 IRs and 51 odorant binding proteins (OBPs) in the screwworm genome ${ }^{30}$. The birth and diversification of these gene families ${ }^{32}$, together with the functional characterization of candidate genes-such as those differentially expressed between sexes, or physiological stages of female-offers the potential to isolate and identify chemicals that are highly effective and selective ${ }^{4}$. Taken together, this work identifies the olfactory similarities and differences between two screwworm species and provides insight into the molecular phylogeny of chemosensation in the screwworm flies.

\section{Results}

Olfactory responses to swormlure constituents. Antennal response of C. hominivorax and C. macellaria to the components of swormlure- $4^{8}$ were measured using the electroantennogram (EAG) technique. In addition, we tested the response of dimethyl trisulfide, a recently described attractive odorant for the screwworm $^{33}$. These compounds elicited robust and highly reproducible EAG responses in both species (Fig. 1). Two compounds, acetic acid and butyric acid, elicited both hyperpolarization and depolarization in both species and therefore were omitted from subsequent statistical analyses. Majority of the antennal responses to tested chemicals-six of the nine-were not significantly different between sexes, or females' mating status. However, antennal response by $C$. hominivorax to two compounds, benzoic acid and phenol, differed between sex and/or mating status (Fig. 2a). Response of unmated females to benzoic acid was smaller than that of males $(p=0.023)$, while the response of unmated females to phenol was larger than that of mated females $(p=0.012)$ (Fig. 2a).

The antennal response of $C$. macellaria to three compounds-2-butanol, dimethyl disulfide and valeric aciddiffered among sex and/or mating status, out of the 9 chemicals tested (Fig. 2b). The response of C. macellaria unmated females and males to 2-butanol was larger than that of mated females $(p=0.023)$, while the response of unmated females to dimethyl disulfide was smaller than that of mated females $(p=0.023)$, and the response to valeric acid was larger in unmated females compared to males $(p=0.028)$ (Fig. 2b). Principal component analysis (PCA) of antennal responses to the nine compounds listed in Fig. 2a and b separated C. hominivorax and C. macellaria by PC1 ( $\mathrm{p} \leq 0.001$, Fig. $2 \mathrm{~d}$ ); however, intraspecies differences based on sex and/or mating status were not obvious in the PCA plot (Fig. 2c). PC1 captured the most variation (39.5\%). The top three PC1 loading 


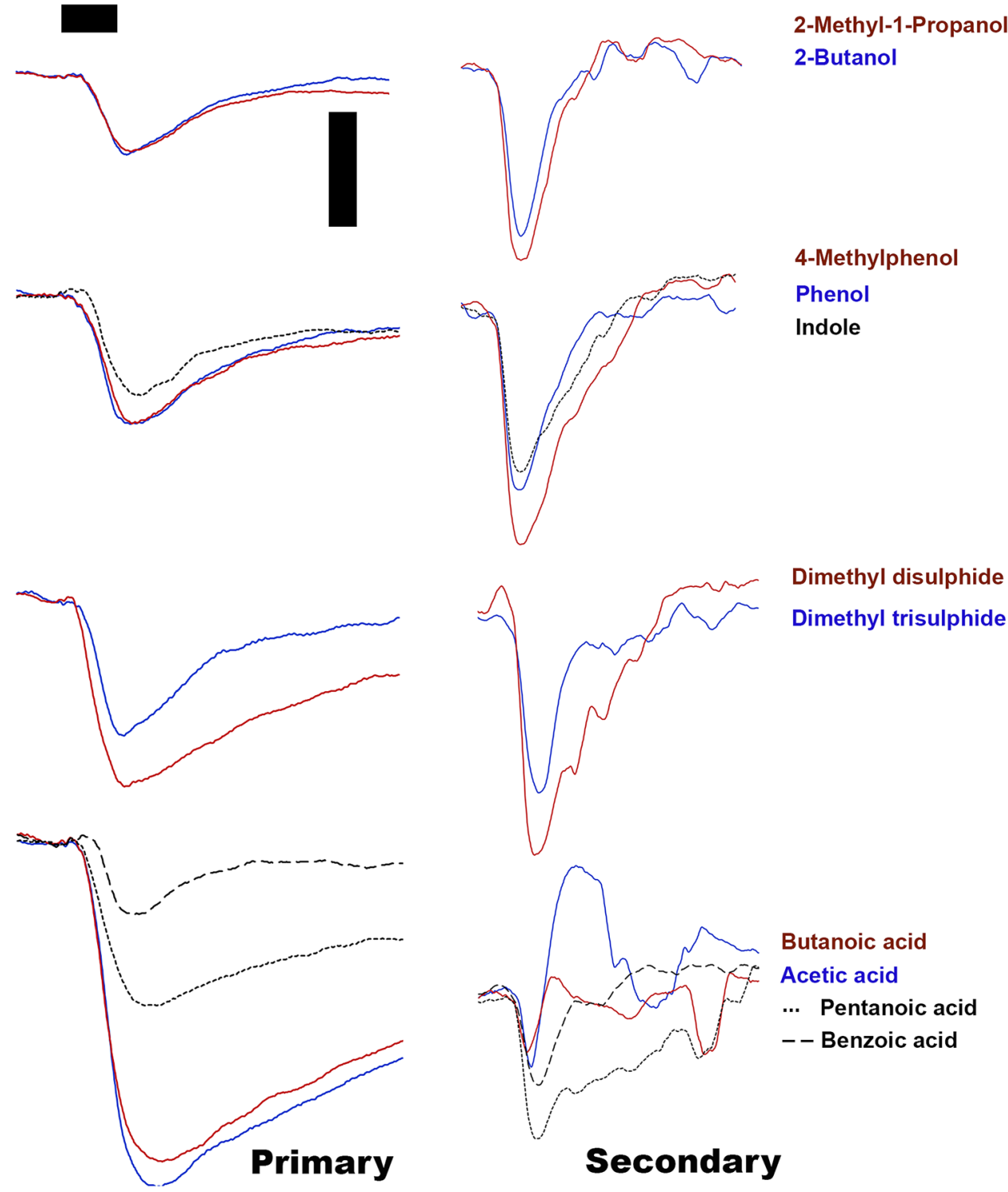

Figure 1. Antennal responses of the primary (left) and secondary screwworm flies to the constituent odorants of swormlure- 4 as measured by electroantennography. Traces are typical EAG signals depicting downward deflections, except acetic acid and butanoic acid traces from primary screwworm flies. Scale bars are $500 \mathrm{~ms}$ (horizontal; also represents the onset of the stimulus delivery) and $1 \mathrm{mV}$ (vertical).

factors (weighing negatively) were 2-methyl-1-propanol, 2-butanol and dimethyl trisulfide, and the top three positively weighing compounds were indole, $p$-cresol and phenol.

Male and female antennal transcriptomes of C. hominivorax. A total of 10,442 genes were expressed in the antennae (Supplemental file 1), of which 114 were annotated as chemosensory genes (55 ORs, 15 GRs, 21 IRs and 23 OBPs). It is worth mentioning that these numbers are lower than those we reported in whole genome annotation $^{30}$, and simply reflect the fact that some of these genes are expressed in other tissues such as legs and mouth parts, and/or at different developmental stages. A total of 85 genes were differentially expressed (DE) between the two sexes (Fig. 3a): 34 were higher in males (male-biased) and 51 were higher in females (femalebiased). Twenty-seven of the 85 differentially expressed genes (31.8\%) were chemosensory genes (16 ORs, 3 IRs and 8 OBPs) (Fig. 3a). We next explored sex-specific differences in chemosensation by conducting principle component analysis (PCA) of all 114 chemosensory genes. PCA based on gene expression of the chemosensory genes showed a clear separation of the sexes by PC1, which explained $49.5 \%$ of the variation (Fig. $3 \mathrm{~b}$ ). Validation by qRT-PCR using independently isolated RNA of six DE ORs-three each male-biased (ChomOr63, ChomOr13 and ChomOr74) and female-biased (ChomOr52, ChomOr62 and ChomOr7)-expression corroborated results based on RNA-seq (Fig. 3c). 

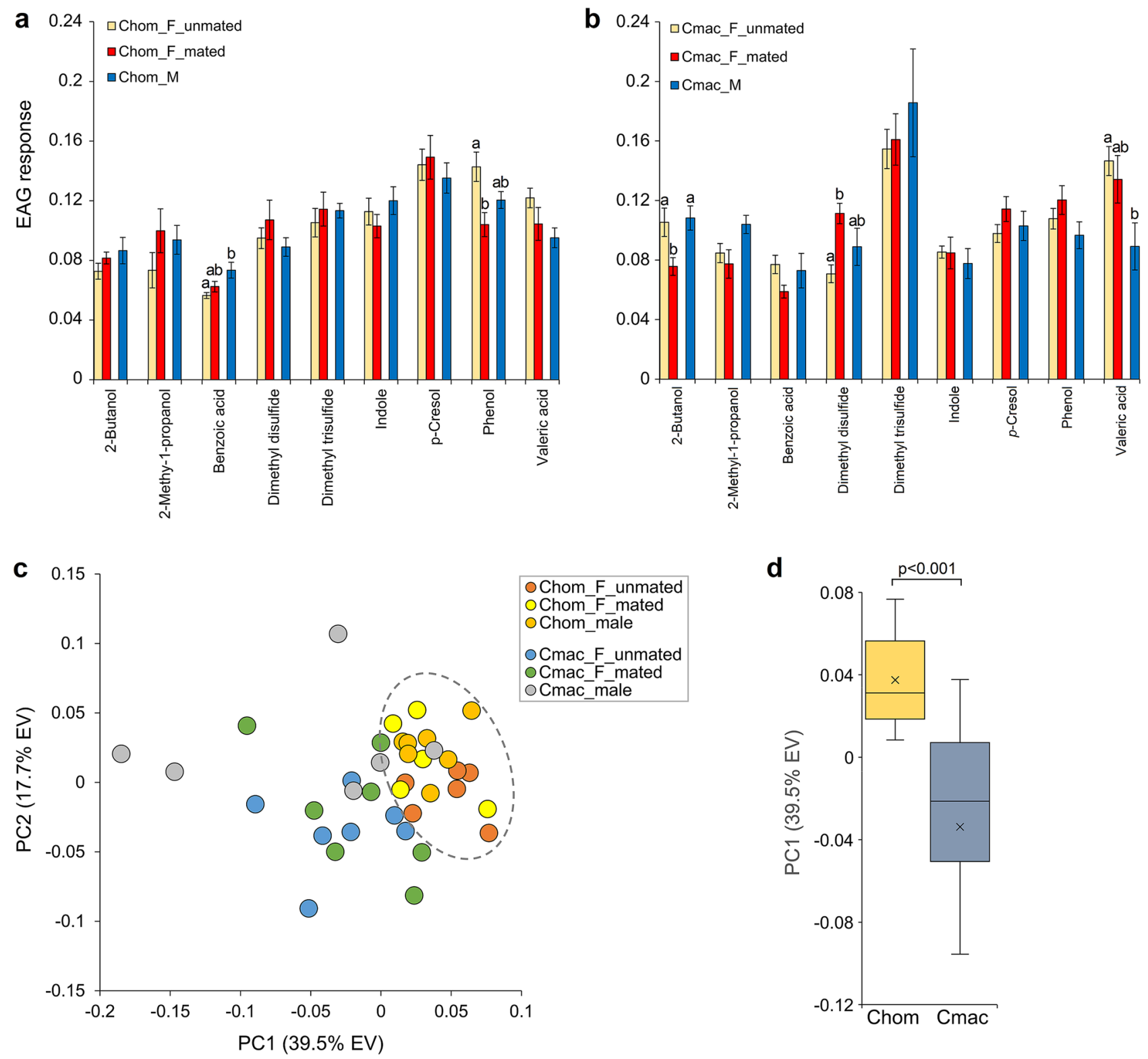

Figure 2. Antennal response by the primary (C. hominivorax) and secondary (C. macellaria) screwworm flies to components of swormlure-4. (a) Benzoic acid elicited a significantly larger response from male C. hominivorax compared to unmated females, while phenol elicited a larger response from unmated females compared to mated females. Significance (indicated by different letters above the columns in $(\mathbf{a}, \mathbf{b})$ based on one-way ANOVA and Tukey's post-hock tests $(p<0.05)$. (b) 2-Butanol elicited a smaller response in mated female $C$. macellaria, while dimethyl disulfide elicited a larger response from mated compared to unmated females. Valeric acid elicited a larger response from unmated females compared to males. (c) Principal component analysis based on the antennal responses largely separated C. hominivorax and C. macellaria. (d) Species were significantly different based on Mann-Whitney rank sum test of PC1 component scores.

A total of 55 ORs were expressed in both sexes, with the exception of Or3, which had no read counts in any of the male replicates. Of the 55 ORs expressed in the antennae, 16 (29.1\%) were DE, with eight displaying femalebiased expression and eight displaying male-biased expression (Fig. 4a). Based on the estimated OR phylogeny, three female-biased and six male-biased ORs were closely related to D. melanogaster ORs (Fig. 5b). Five ORs with male-biased expression (ChomOr13, ChomOr65, ChomOr73, ChomOr74 and ChomOr78) are in a clade with DmelOr67d, the cis-vaccenyl acetate pheromone receptor ${ }^{34,35}$ (Fig. 4b,c).

Three of the 21 IRs expressed in the antennae were DE between sexes, all being female-biased (Fig. 5a). The estimated IR phylogeny suggests that these are closely related to their namesake in D. melanogaster (Fig. 5b). In D. melanogaster, Ir75a detects several carboxylic acids (acetic, butyric and propionic) ${ }^{40}$, while $\operatorname{Ir} 76 a$ detects amines such as pyrrolidine and phenylethylamine ${ }^{41}$ (Fig. $5 \mathrm{c}$ ). The types of IRs expressed in C. hominivorax were similar to those expressed in D. melanogaster antennae, with the following exceptions. ChomIr31a, ChomIr60a and ChomIr68a were not expressed in C. hominivorax but are commonly expressed in D. melanogaster, while 

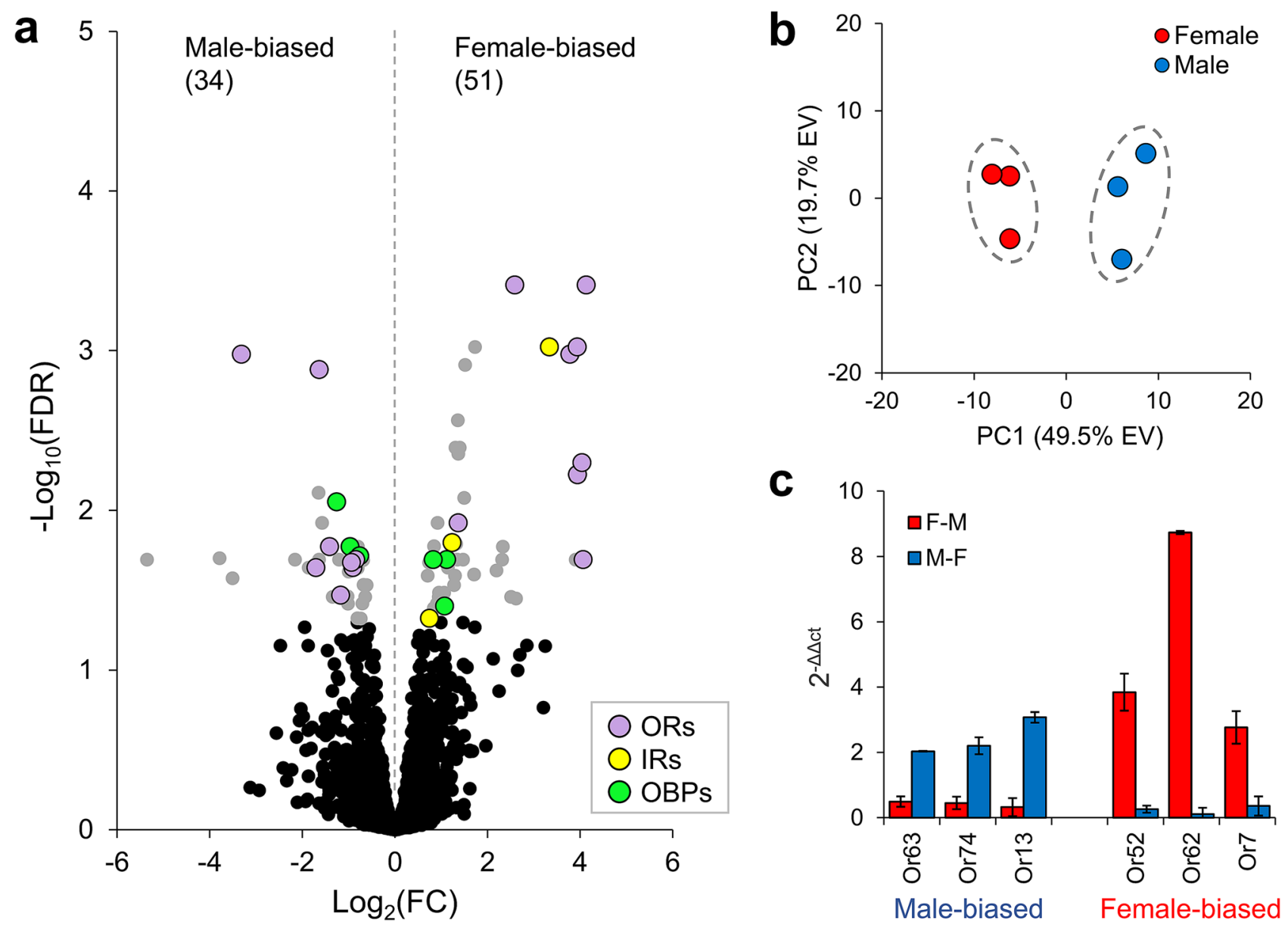

Figure 3. Quantitative analysis of the antennal transcriptome in male and female $C$. hominivorax. (a) A total of 10,442 genes were expressed in the antennae, of which 85 were differentially expressed (DE) between sexes (FDR corrected $p<0.05)$. Thirty-four genes showed male-biased expression and 51 showed female biased expression. Of the 85 DE genes, 27 were chemosensory genes (16 ORs, 0 GRs, 3 IRs and 8 OBPs). (b) Multivariate analysis was used to explore sex-specific differences in 114 chemosensory genes expressed in the antennae. PC1 explained $49.5 \%$ of the variation and grouped the samples according to sex. (c) RT-qPCR validation of six olfactory receptor genes differentially expressed between male and female $C$. hominivorax antennae. Data is the average of the biological replicates, and error bars illustrate standard error of the mean (SEM). Expression levels of each of the six genes were significantly different $(\mathrm{p}<0.05)$.

ChomIr10a was expressed in C. hominivorax but is not typically expressed in D. melanogaster antennae ${ }^{42}$. Of the OBPs expressed in the antennae, six were DE between the sexes, with three being female-biased and three being male-biased (Fig. 6a). Five of these are closely related to D. melanogaster OBPs based on the estimated phylogeny (Fig. 6b). Thus far, a role in olfaction has been described only for DmelObp49a, where it was shown to be associated with bitter taste inhibition to sugar feeding ${ }^{43}$ (Fig. 6c).

None of the 15 GRs expressed in C. hominivorax antennae were DE between sexes. The antennal GRs included three putative $\mathrm{CO}_{2}$ receptors, of which two (Chom $\mathrm{Gr} 2$ and Chom $\mathrm{Gr} 3$ ) were among the most highly expressed GRs, while the third (Chom Gr1) was expressed at a much lower level (Fig. S1a,b). All six putative sugar receptors (ChomGr4-Gr9) were expressed in the antennae (Fig. S1a,b). The estimated GR phylogeny suggests ChomGr25 is closely related to DmelGr57a (Fig. S1b), which is associated with bitter taste in D. melanogaster ${ }^{45}$. These results are similar to findings in D. melanogaster in which two $\mathrm{CO}_{2}$ receptors and five sugar receptors were expressed in the antennae ${ }^{46}$. Function of the remaining five GRs is difficult to predict due to their divergence from $D$. melanogaster GRs.

\section{Discussion}

We utilized a multifaceted approach to address knowledge gaps in screwworm fly olfactory physiology, with the aim of developing more effective and specific attractants for screwworm fly control strategies. This was the first study of its kind in which olfactory responses of mated and unmated female C. hominivorax or C. macellaria were compared. Furthermore, we report for the first time quantitative analysis of the antennal transcriptome in male and female C. hominivorax.

Antennal responses of mated and unmated female C. hominivorax to swormlure-4 constituents were similar, except for phenol, which elicited greater EAG signal in unmated females. None of the tested chemicals elicited a 
a

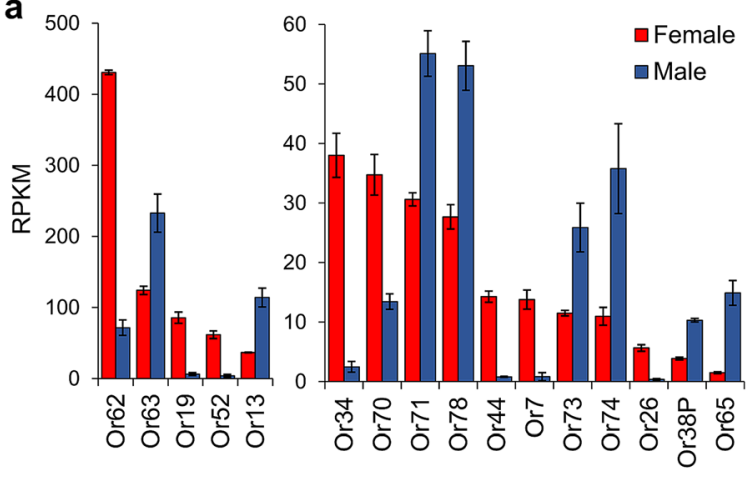

\begin{tabular}{llll}
\hline Chom OR & Bias & $\begin{array}{l}\text { Dmel } \\
\text { ortholog }\end{array}$ & Function in D. melanogaster \\
\hline $\begin{array}{l}\text { Or13, Or65, } \\
\text { Or73, Or74, } \\
\text { Or78 }\end{array}$ & male & Or67d & Response to cis-vaccenyl acetate \\
Or62 & female & Or88a & $\begin{array}{l}\text { Attraction to methyl laurate, methyl } \\
\text { myristate and methyl palmitate }\end{array}$ \\
Or63 & male & Or85f & $\begin{array}{l}\text { Avoidance of the parasitoid volatiles } \\
\text { actinidine and nepetalactol }\end{array}$ \\
Or19 & female & Or7a & Aggregation response to 9-tricosene \\
\hline
\end{tabular}

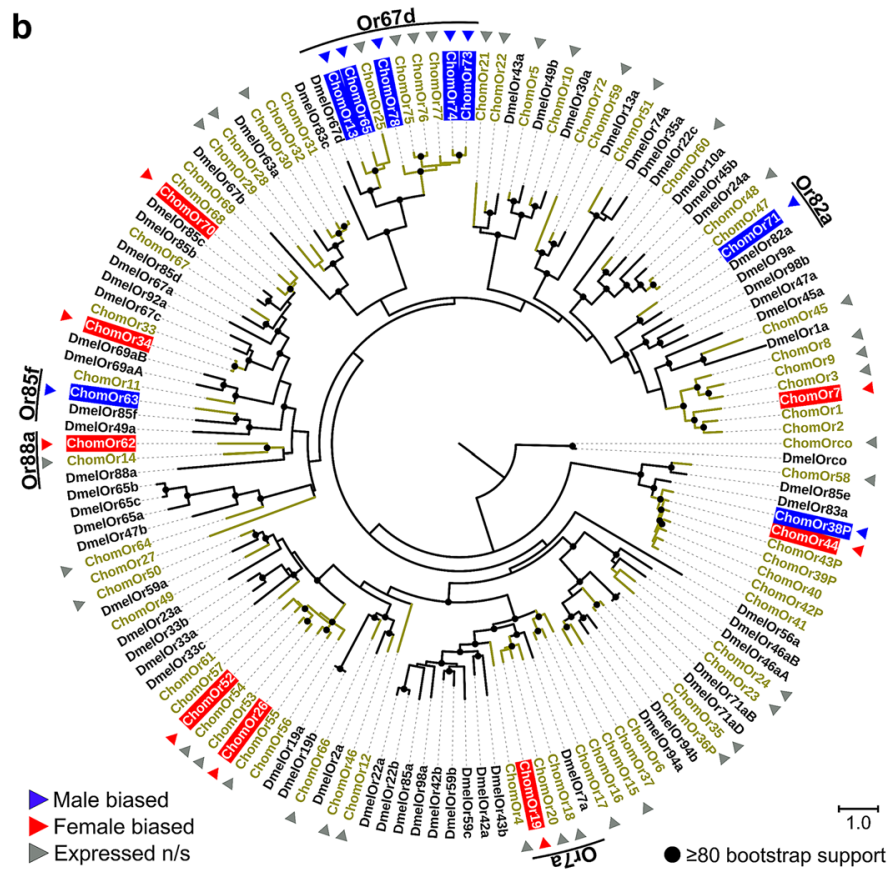

Figure 4. Sex-biased expression of ORs in C. hominivorax antennae. (a) Fifty-five ORs were expressed in the antennae, of which 16 were differentially expressed between males and females, 8 each in females and males. Differential expression based on FDR adjusted $\mathrm{p}<0.05$. (b) Nine $C$. hominivorax ORs are closely related to $D$. melanogaster ORs based on the estimated OR phylogeny. Figures were generated using the interactive Tree of Life (iTOL) v4 software ${ }^{36}$ (https://itol.embl.de/). (c) Five male-biased ORs are closely related to the cis-vaccenyl acetate (cVA) pheromone receptor (Or67d) in D. melanogaster ${ }^{34,35}$, while one (Or63) is closely related to Or85f, which has been implicated in avoidance of the parasitoid volatiles actinidine and nepetalactol ${ }^{37}$. Two of the female-biased ORs are closely related to the 9-tricosene (Or7a), and methyl laurate, methyl myristate and methyl palmitate (Or88a) receptors in D. melanogaster ${ }^{38,39}$, all of which are implicated as fly pheromones.

a

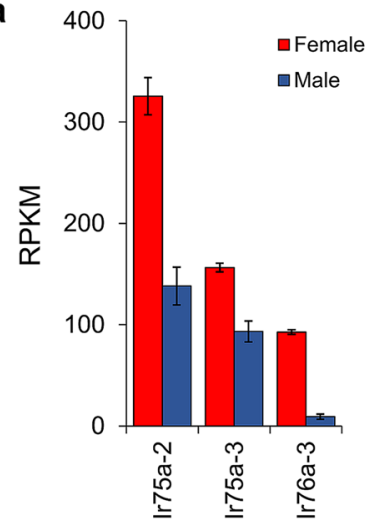

C

\begin{tabular}{|c|c|c|c|}
\hline Chom IR & Bias & $\begin{array}{l}\text { Dmel } \\
\text { ortholog }\end{array}$ & Function in D. melanogaster \\
\hline $\begin{array}{l}\text { Ir75a-2, } \\
\text { Ir75a-3 }\end{array}$ & female & Ir75a & $\begin{array}{l}\text { Olfactory response to propionic, } \\
\text { butyric and acetic acids }\end{array}$ \\
\hline Ir76a-3 & female & Ir76a & $\begin{array}{l}\text { Olfactory response to } \\
\text { phenylethylamine }\end{array}$ \\
\hline
\end{tabular}

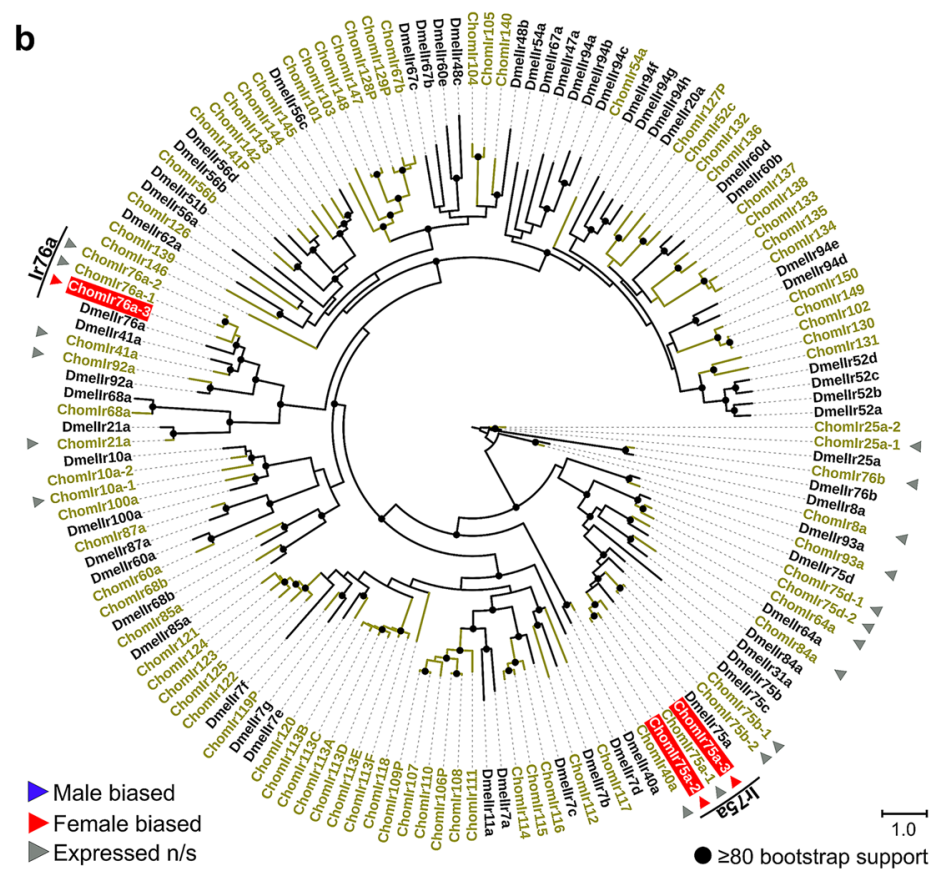

Figure 5. Sex-biased expression of IRs in C. hominivorax antennae. (a) Twenty-one IRs were expressed in the antennae, of which three were differentially expressed (DE) between sexes, all with higher expression in females. Differential expression based on FDR adjusted $p<0.05$. (b) The three DE IRs are related to two D. melanogaster receptors based on the IR phylogeny. Figures were generated using the interactive Tree of Life (iTOL) v4 software $^{36}$ (https://itol.embl.de/). (c) In D. melanogaster, Ir75a confers an olfactory response to propionic, butyric and acetic acids ${ }^{40}$, while Ir76a elicits an olfactory response to phenylethylamine ${ }^{41}$. 
a

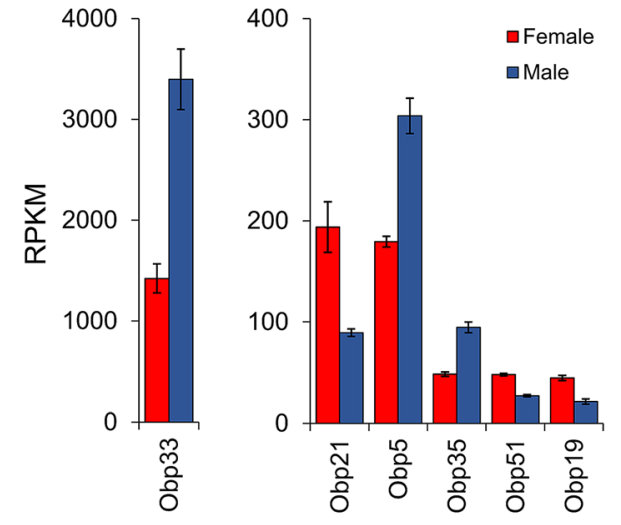

C

\begin{tabular}{llll}
\hline Chom OBP & Bias & $\begin{array}{l}\text { Dmel } \\
\text { ortholog }\end{array}$ & Function in $\boldsymbol{D}$. melanogaster \\
\hline $\begin{array}{l}\text { Obp33, } \\
\text { Obp35 }\end{array}$ & male & Obp56a & $\begin{array}{l}\text { Unknown. Downregulated in mated } \\
\text { female Drosophila and Bactrocera tryoni }\end{array}$ \\
$\begin{array}{llll}\text { Obp19, } \\
\text { Obp21 }\end{array}$ & female & Obp19b & Taste perception of amino acids \\
\hline
\end{tabular}

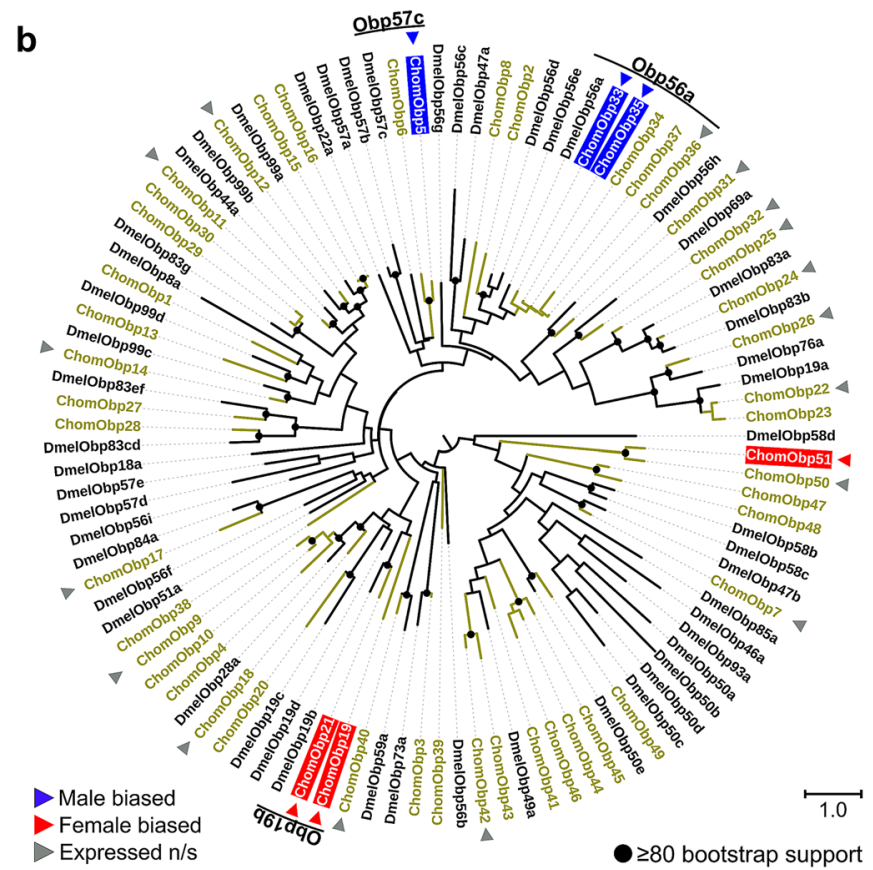

Figure 6. Sex-biased expression of OBPs in C. hominivorax antennae. (a) Twenty-three OBPs were expressed in the antennae, of which six were DE between sexes, three with male-biased expression and three with femalebiased expression. Differential expression based on FDR adjusted $\mathrm{p}<0.05$. (b) Of the six DE OBPs, five are related to $D$. melanogaster proteins based on the estimated OBP phylogeny. Figures were generated using the interactive Tree of Life (iTOL) v4 software ${ }^{36}$ (https://itol.embl.de/). (c) In D. melanogaster, Obp19b is involved with taste perception of amino acids ${ }^{4}$. The chemosensory role of the remaining DE OBPs is largely unknown.

greater response in mated females. This is somewhat unexpected since the swormlure constituents were mostly derived from the analysis of bovine blood and odors associated with decomposition of meat protein and fatty acids representing an oviposition substrate for screwworm flies ${ }^{15}$. In C. macellaria, however, dimethyl disulfide elicited a larger physiological response in mated flies compared to unmated females. An additional use for a lure attracting primarily mated females, other than surveillance, is the potential for the development of a lethal oviposition trap for population control. The utility of such a trap has been demonstrated for mosquito species ${ }^{47}$, and remains to be for explored for control of screwworm.

There were no significant differences in the antennal responses to the tested constituents when males were compared with mated and unmated female C. hominivorax or C. macellaria. This is in contrast to a report where $p$-cresol (4-methylphenol) and dimethyl disulfide elicited a greater physiological response in males ${ }^{48}$. Field studies have differed in their proportion of males and females collected in traps baited with swormlure-4, with studies ranging from no sex bias $^{49}$ to strong female-bias ${ }^{8,29,50}$. It was demonstrated during the development of the first synthetic lure (swormlure) that attraction was greater to the blend than to the individual constituents ${ }^{15}$, therefore, it is possible that the blend (swormlure-4) elicits a sex biased response while the individual constituents do not.

Multivariate analysis (PCA) suggests C. hominivorax and C. macellaria differed in their antennal responses to the swormlure constituents, even though sex or physiological state (virgin vs. gravid) differences were not that apparent within a species. However, this should be interpreted with caution: EAG studies for C. macellaria were performed in the laboratory at the University of Kentucky wherein optimum recording condition, such as stable temperature, humidity and noise-free electrical contacts are observed. Antennal recordings of C. hominivorax conducted at COPEG didn't have all these optimal conditions. Consequently, we avoided direct comparison of EAG responses of $C$. hominivorax with C. macellaria. Due to their close evolutionary relationship yet different oviposition substrates preference (live animal wounds vs carrion or necrotic tissue), comparative chemical ecology and molecular olfaction studies of $C$. hominivorax and C. macellaria could improve the species-specificity of synthetic attractants for the screwworm fly and yield insights into the evolution of parasitism in Cochliomyia in the future.

In contrast to our EAG analysis of $C$. hominivorax in which there were no major sex-specific differences in antennal responses elicited by the tested chemicals, antennal transcriptome analysis revealed $29 \%$ of the expressed ORs as sex-biased. The simplest explanation for the disparity between EAG responses and gene expression analysis is that the EAGs are only proximate summation of the individual olfactory receptor neuron (ORN) responses $^{51}$, and do not faithfully reflect the response of all chemosensory genes. Recent evidence also suggests variation in the transcriptional profiles of the chemosensory genes in natural mosquito populations ${ }^{52}$. Another likely explanation for absence of any sex-based correlation between the transcriptional profiles (Fig. 3) and EAG responses (Fig. 2) is that the ORs that are major contributors for the sexually dimorphic transcription profiles do not significantly contribute to the EAG amplitudes measured for the swormlure-4 constituents tested here. 
Indeed conventional EAG measurements are largely the summed responses from basiconic sensilla, and underrepresent the contribution of trichoid sensilla ${ }^{51,53}$.

Our phylogenetic analysis of the olfactory genes groups nine of the 16 sex-biased $C$. hominivorax ORs with D. melanogaster receptors associated with pheromone sensing ${ }^{35,38,39}$. In Drosophila, trichoid sensilla respond to pheromone related odors, whereas most of the food/host odors are detected by basiconic sensilla ${ }^{54,55}$. ChomOr63 (male-biased) is closely related to DmelOr85f, which was shown to be associated with innate avoidance of actinidine and nepetalactol, volatiles associated with the Drosophila parasitoids in the genus Leptopilina ${ }^{37}$ (Fig. 4b,c). ChomOr19 and ChomOr62 (female-biased) are closely related to DmelOr7a and DmelOr88a, respectively (Fig. 4b). DmelOr7a was shown to be necessary for aggregation and oviposition site selection via 9-tricosine signaling ${ }^{39}$, while DmelOr88a was required for short-range attraction of both sexes to the fly-produced odors methyl laurate, methyl myristate and methyl palmitate ${ }^{38}$ (Fig. 4c). The remaining seven sex biased ORs had either no close phylogenetic relationship or their function has not yet been well characterized in D. melanogaster. Previous studies have established a role for chemical sexual communication in C. hominivorax, implicating male-produced ${ }^{56}$ and female-produced ${ }^{57,58}$ substrates such as cuticular hydrocarbons (CHCs). However, despite the identification of several $\mathrm{CHCs}^{59-61}$, their behavioral significance has yet to be realized. With the availability screwworm genome $e^{30}$, and specifically the development of genetic knockout techniques in these flies such as CRISPR/Cas9 ${ }^{62}$, we aim to understand these behaviors driven by chemosensory genes. Identification of the ligands for the male- and female-biased receptors we describe here could identify chemicals for the development of sex-specific attractants.

The contribution of antennal IR expression on the EAG responses is unclear. These genes are primarily expressed in coeloconic sensilla ${ }^{41,42}$. Two of the three female-biased and highly-expressed IRs in the C. hominivorax antennal transcriptome (ChomIr75a-2 and ChomIr75a-3) are closely related to DmelIr75a, which responds to the short chain carboxylic acids ${ }^{40}$. Electroantennograms represent an approximate global olfactory responses from the antenna depicting the overall response dynamics and amplitude resulting from multiple ORNs expressing different families of chemosensory receptor genes, such as ORs, IRs or GRs ${ }^{63,64}$. As we noted earlier, acetic acid and butyric acid induced occasional hyperpolarizations. Short chain carboxylic acids, especially at high doses, often elicit upward deflection in EAG recordings across a range of insects ${ }^{65}$, and this has been ascribed to the acidity of these compounds and/or sometimes, as an artifact electrode potentials resulting from interaction of acids with the electrodes ${ }^{66}$. Our conclusion of the acid induced hyperpolarization, based on multiple measurements across both sexs and species, is that the measured signals are not artifacts.

Monitoring for the presence of $C$. hominivorax is an important component of screwworm control and eradication programs ${ }^{67}$. Though various measures are utilized for monitoring, synthetic attractants (e.g. swormlure) are particularly appealing due to the ease and expense at which they can be employed compared to alternative attractants, such as rotten beef liver. However, gaps in our knowledge have prevented advancement of synthetic attractant strategies for the screwworm fly. Improvement in species specificity and the development of a malespecific and gravid-female specific attractants is highly desirable. Here, we used a multifaceted approach in which we integrated neurophysiology, molecular and phylogenetic methods to gain a better understanding of C. hominivorax biology, toward the development of novel attractants for control of screwworm. We are in the process of functional characterization of the promising chemosensory genes we identified here based on the differential expression pattern and their phylogenetic relationship to the D. melanogaster orthologues that have been fully characterized. Prospecting the cognate ligands from the natural milieus such as open wounds and conspecifics will lead to the isolation and identification of chemostimuli that will be tested for the behavioral response, and eventually as odor baits in the surveillance traps.

\section{Methods}

Flies. The J06 strain of $C$. hominivorax was used for antennal transcriptome analysis and electroantennography (EAG). The J06 wild type strain of $C$. hominivorax was originally collected in Jamaica in 2006 , and this strain was being used at the COPEG in Panama for SIT release at the time of the study.Flies were reared at the COPEG biosecurity plant in Panama using methods described previously ${ }^{68}$. C. macellaria used for EAG were originally collected in 2011 from a pig carcass located at the North Carolina State University (NCSU) Poultry field laboratory in Raleigh, North Carolina $(35.724 \mathrm{~N},-76.687 \mathrm{~W})$, and are maintained at North Carolina State University at $27^{\circ} \mathrm{C}$ with 16:8 light dark cycle. Adult flies were provisioned with granulated sugar and water ad libitum. Weekly, adult fly cages were provided with $10 \mathrm{~g}$ of raw beef liver as a protein source to optimize ovary development. Two-week-old flies were provided a cup containing $10 \mathrm{~g}$ of raw liver loosely covered with a moist paper towel to encourage oviposition. Collected eggs were partitioned into pea sized $(10 \mathrm{~mm})$ allotments for transfer to the prepared larval medium consisting of $750 \mathrm{ml}$ dry Purina Cat Chow Complete (Nestle Purina PetCare Company, St. Louis, MO) soaked in $400 \mathrm{ml}$ of water. In a well-ventilated room, the larval medium bucket was placed on a rack at a $35^{\circ}$ angle, the eggs added and the bucket covered with a screened lid. Pupae were harvested in 7 to 8 days.

EAG recordings. Mounting of $C$. hominivorax for EAG was conducted by excising the head of the fly (3-6 days post-eclosion) with microscissors and placing it on an antenna holder (Syntech, Germany) with the base of the head on one side and the tip of both antennae contacting the other. To improve electrical conduction and reduce desiccation, Spectra 360 Electrode Gel (Parker Laboratories) was applied at the contacts between the holder and the fly (base of head and tip of antennae). We note that due to biosecurity measures, these recordings could only be conducted inside the COPEG production facility, which is not optimal for fine electrophysiological measurements. Mounting of C. macellaria for EAG was similar to fruit flies recordings we routinely employ ${ }^{69}$ with a few modifications. A fly (3-8 days post-eclosion) was inserted in a $1000 \mu$ l pipette tip (USA Scientific Inc.) 
with approximately one-third of the head protruding from the tip. The tips of both antennae were placed in a $1 \mathrm{~mm}$ borosilicate capillary (World Precision Instruments, USA) containing sensillum lymph ringer ${ }^{65}$, while a ground was placed into the eye. Signals were amplified and recorded using an IDAC2-USB box (Syntech) with a $0.05 \mathrm{~Hz}$ low cutoff. Each recording was a total of $10 \mathrm{~s}$ in length with a set $1 \mathrm{~s}$ pre-trigger. A CS-55 Stimulus Controller (Syntech) was used to supply a charcoal filtered and humidified continuous air stream $(0.8 \mathrm{l} / \mathrm{min})$ delivered via a Teflon tube. A stimulus pulse $(0.8 \mathrm{l} / \mathrm{min})$ was added to the air stream for $0.5 \mathrm{~s}$. Recordings were analyzed with EAG Pro version 1.1 software (Syntech).

Chemicals used for stimulus and solvent were of high purity: 1-octen-3-ol (98\% purity) 2-butanol (analytical standard), 2-methyl-1-propanol (analytical standard), acetic acid (99.5\%), benzoic acid (analytical standard), butyric acid (analytical standard), dimethyl disulfide (analytical standard), dimethyl trisulfide (98\%), indole (98\%), p-cresol ( $\geq 99 \%)$, phenol ( $\geq 99 \%)$ and valeric acid (analytical standard). Chemicals were dissolved in dichloromethane (DCM $\geq 98 \%)$ to make a stock solution of $100 \mathrm{mg} / \mathrm{ml}(-1)$. A 1:10 dilution (-2) of the stock solution in DCM was used as stimulus. A $20 \mu \mathrm{l}$ of stimulus solution was loaded onto a filter paper strip. After allowing the solvent to evaporate for $10-15 \mathrm{~s}$, the strip was placed in a $5 \mathrm{ml}$ polypropylene syringe for delivery onto the EAG preparation. Each recording was first baseline normalized, then smoothed with a $200 \mathrm{~ms}$ running average, amplitude determined and the value of the appropriate blank (solvent) control subtracted.

The antennal response for each compound is represented as the proportion of the summed responses of each individual to all compounds. Statistical tests for difference in antennal response between sexes and/or physiological state (virgin and gravid), and principal component analysis (PCA) were conducted using SigmaPlot v14.0. Data were initially assessed and passed tests for normality (Shapiro-Wilk) and equal variance (Brown-Forsythe) prior to implementing a one-way ANOVA and Tukey post-hoc test (when ANOVA p-value <0.05). Principal component analysis was conducted with the covariance method using the average eigenvalue for component selection. The Mann-Whitney rank sum test was used to test for difference between principal component scores for PC1 in Fig. 2 d.

Antennal transcriptome analysis. The methods used for extraction and quantification of total RNA, and preparation of cDNA libraries are described in Scott et al. ${ }^{30}$. In brief, antennal RNA was extracted from frozen samples, and RNA integrity, purity, and concentration were assessed using an Agilent 2100 Bioanalyzer. Purification of mRNA was performed using the oligo-dT beads provided in the NEBNExt Poly (A) mRNA Magnetic Isolation Module. Complementary DNA libraries for Illumina sequencing were constructed using the NEBNext Ultra Directional RNA Library Prep Kit (NEB) and NEBNext Mulitplex Oligos for Illumina (NEB) using the manufacturer-specified protocol. The amplified library fragments were purified and checked for quality and final concentration using an Agilent 2200 Tapestation with a High Sensitivity DNA chip (Agilent Technologies, USA) and a Qubit fluorometer (ThermoFisher, USA). The quantified cDNA libraries were pooled in equimolar amounts for clustering and sequencing on an Illumina HiSeq 2500 DNA sequencer, utilizing a 125 bp single-end sequencing reagent kit (Illumina, USA).

Sequencing depth ranged from 8.74 to 13.06 million mapped reads per library $($ mean $=10.45)$. Sequence reads were mapped to the $C$. hominivorax genome assembly using Hisat 2 v2.1.0 $0^{70}$. Read counts were obtained using HTSeq-count (union mode) in HTSeq v0.11.2 $2^{71}$. The quasi-likelihood F-test and false discovery rate (fdr) correction for multiple tests in EdgeR was used for differential expression ${ }^{72,73}$. Data were converted from CPM (counts per million reads) to RPKM (reads per kilo base per million mapped reads) following statistical analysis. SigmaPlot v14.0 was used for PCA of chemosensory gene expression (RPKM) using the correlation method and the average eigenvalue for component selection.

RT-qPCR validation of candidate $C$. hominivorax genes. RNA was isolated following the Direct-zol protocol (Zymo Research, Irvine, CA) from five pairs of male and female adult fly antenna that were shipped from COPEG, Panama in RNA-shield (Zymo Research, Irvine, CA). The quantity and quality of the isolated RNA was assessed using a NanoDrop. First-strand cDNA was synthesized from $300 \mathrm{ng}$ of total RNA per sample using the Superscript II protocol (Thermo Fisher, Carlsbad, CA). cDNA for each sample was diluted to $20 \mathrm{ng} / \mu \mathrm{l}$ for subsequent qPCR reactions. Actin and RPS17 served as endogenous control genes for qPCR since these were shown to be the top and most stably expressed genes within the members of Calliphoridae ${ }^{74}$. Each $10 \mu \mathrm{l}$ PCR reaction included $2 \mu \mathrm{l}$ of cDNA template $(20 \mathrm{ng} / \mu \mathrm{l}), 5 \mu \mathrm{l}$ of SYBR GRN master mix, $1 \mu \mathrm{l}$ of primer $(0.5 \mu \mathrm{l} \mathrm{F}$ and $0.5 \mu \mathrm{R})$ and $2 \mu \mathrm{l}$ of sterile water. PCR cycling conditions were $95^{\circ} \mathrm{C}(3 \mathrm{~min})$ and 40 cycles of $95^{\circ} \mathrm{C}(10 \mathrm{~s})$ and $60^{\circ} \mathrm{C}$ for $30 \mathrm{~s}$. For each gene, a standard curve was derived to assess the amplification efficiency of the primers, melting curve to assess the specificity of the primers and included three biological replicates. Differential expression analysis was based on the well establish method ${ }^{75}$.

Phylogenetic analysis of chemosensory genes. Phylogenetic relationships among C. hominivorax ${ }^{30}$ and D. melanogaster ${ }^{40,76,77}$ chemosensory genes were estimated by first conducting a multiple protein alignment for each gene family using default parameters in ClustalX v2.17. Alignments were visually inspected and then used for maximum likelihood estimation in RAxML v8.2.11 with the PROTGAMMAAUTO model option and 500 bootstrap replications ${ }^{79}$. Figures were generated using the interactive Tree of Life (iTOL) v4 software ${ }^{36}$ (https ://itol.embl.de/). 
Received: 27 August 2020; Accepted: 10 November 2020

Published online: 27 November 2020

\section{References}

1. Baumhover, A. H. Eradication of the screwworm fly: an agent of myiasis. JAMA 196, 240-248 (1966).

2. Bushland, R. Screwworm research and eradication. Bull. ESA 21, 23-26 (1975).

3. Scott, M. J., Concha, C., Welch, J. B., Phillips, P. L. \& Skoda, S. R. Review of research advances in the screwworm eradication program over the past 25 years. Entomol. Exp. Appl. 164, 226-236 (2017).

4. Carey, A. F. \& Carlson, J. R. Insect olfaction from model systems to disease control. Proc. Natl. Acad. Sci. 108, 12987-12995 (2011).

5. Syed, Z. Chemical ecology and olfaction in arthropod vectors of diseases. Curr. Opin. Insect Sci. 10, 83-89 (2015).

6. LeBlanc, H. N. \& Logan, J. G. Current Concepts in Forensic Entomology 205-221 (Springer, New York, 2009).

7. Pickett, J. A. et al. Chemical ecology of animal and human pathogen vectors in a changing global climate. J. Chem. Ecol. 36, 113-121 (2010).

8. Mackley, J. W. \& Brown, H. E. Swormlure-4: a new formulation of the swormlure-2 mixture as an attractant for adult screwworms, Cochliomyia hominivorax (Diptera: Calliphoridae). J. Econ. Entomol. 77, 1264-1268 (1984).

9. Parker, F. D. \& Welch, J. B. Monitoring adult populations of the screwworm (Diptera: Calliphoridae) with feeding stations baited with liver. J. Econ. Entomol. 85, 1740-1753 (1992).

10. Bishopp, F. Flytraps and their operation. US Dep. Agric. 734, 1 (1937).

11. Laake, E., Cushing, E. C. \& Parish, H. Biology of the primary screw worm fly, Cochliomyia americana, and a comparison of its stages with those of C. macellaria. (United States Department of Agriculture, Economic Research Service, 1936).

12. DeVaney, J. A., Eddy, G. W., Ellis, E. M. \& Harrington, R. Attractancy of inoculated and incubated bovine blood fractions to screwworm flies (Diptera-Calliphoridae): role of bacteria. J. Med. Entomol. 10, 591-595. https://doi.org/10.1093/jmedent/10.6.591 (1973).

13. Hammack, L. \& Holt, G. G. Responses of gravid screwworm flies, Cochliomyia hominivorax, to whole wounds, wound fluid, and a standard blood attractant in olfactometer tests. J. Chem. Ecol. 9, 913-922. https://doi.org/10.1007/bf00987814 (1983).

14. Grabbe, R. \& Turner, J. Screwworm attractants: isolation and identification of organic compounds from bacterially inoculated and incubated blood. Folia Entomol. Mex 25, 120-121 (1973).

15. Jones, C. M., Oehler, D. D., Snow, W. J. \& Grabbe, R. R. A chemical attractant for screwworm flies. J. Econ. Entomol. 69, 389-391 (1976).

16. Coppedge, J. R., Ahrens, E., Goodenough, J. L., Guillot, F. S. \& Snow, J. W. Field comparisons of liver and a new chemical mixture as attractants for the screwworm fly. Environ. Entomol. 6, 66-68 (1977).

17. Snow, J. W., Coppedge, J. R., Broce, A. B., Goodenough, J. L. \& Brown, H. E. Swormlure: development and use in detection and suppression systems for adult screwworm (Diptera: Calliphoridae). Bull. ESA 28, 277-285 (1982).

18. Cork, A. Identification of electrophysiologically-active compounds for New World screwworm, Cochliomyia hominivorax, in larval wound fluid. Med. Vet. Entomol. 8, 151-159 (1994).

19. Cork, A. \& Hall, M. Development of an odour-baited target for female New World screwworm, Cochliomyia hominivorax: studies with host baits and synthetic wound fluids. Med. Vet. Entomol. 21, 85-92 (2007).

20. Skoda, S. R., Phillips, P. L. \& Welch, J. B. Screwworm (Diptera: Calliphoridae) in the United States: response to and elimination of the 2016-2017 outbreak in Florida. J. Med. Entomol. 55, 777-786 (2018).

21. Gadenne, C., Barrozo, R. B. \& Anton, S. Annual review of entomology. Annu. Rev. Entomol. 61, 317-333 (2016).

22. Carey, J. R. et al. Age-specific and lifetime behavior patterns in Drosophila melanogaster and the Mediterranean fruit fly, Ceratitis capitata. Exp. Gerontol. 41, 93-97. https://doi.org/10.1016/j.exger.2005.09.014 (2006).

23. Tasnin, M. S., Merkel, K. \& Clarke, A. R. Effects of advanced age on olfactory response of male and female Queensland fruit fly, Bactrocera tryoni (Froggatt)(Diptera: Tephritidae). J. Insect Physiol. 122, 104024 (2020).

24. Saveer, A. M. et al. Floral to green: mating switches moth olfactory coding and preference. Proc. R. Soc. B 279, 2314-2322 (2012).

25. Foster, W. \& Takken, W. Nectar-related vs. human-related volatiles: behavioural response and choice by female and male Anopheles gambiae (Diptera: Culicidae) between emergence and first feeding. Bull. Entomol. Res. 94, 145-157 (2004).

26. Broce, A. Sexual behavior of screwworm flies stimulated by swormlure-2. Ann. Entomol. Soc. Am. 73, 386-389 (1980).

27. Hammack, L., Bromel, M., Duh, F. \& Gassner, G. Reproductive factors affecting responses of the screwworm fly, Cochliomyia hominivorax (Diptera: Calliphoridae), to an attractant of bacterial origin. Ann. Entomol. Soc. Am. 80, 775-780 (1987).

28. Guillot, F., Coppedge, J., Goodenough, J., Adam, T. \& Ahrens, E. Behavior and reproductive status of native female screwworms attracted to a host. Ann. Entomol. Soc. Am. 70, 588-590 (1977).

29. Welch, J. B. Effect of trap placement on detection of Cochliomyia hominivorax (Diptera: Calliphoridae). J. Econ. Entomol. 81, 241-245 (1988).

30. Scott, M. J. et al. Genomic analyses of a livestock pest, the New World screwworm, find potential targets for genetic control programs. Commun. Biol. 3, 424. https://doi.org/10.1038/s42003-020-01152-4 (2020).

31. Robertson, H. M. Molecular evolution of the major arthropod chemoreceptor gene families. Annu. Rev. Entomol. 64, 227-242 (2019).

32. Benton, R. Multigene family evolution: perspectives from insect chemoreceptors. Trends Ecol. Evol. 30, 590-600 (2015).

33. Zhu, J. J. et al. Semiochemicals released from five bacteria identified from animal wounds infested by primary screwworms and their effects on fly behavioral activity. PLoS ONE 12, e0179090 (2017).

34. Ha, T. S. \& Smith, D. P. A pheromone receptor mediates 11-cis-vaccenyl acetate-induced responses in Drosophila. J. Neurosci. 26, $8727-8733(2006)$

35. Kurtovic, A., Widmer, A. \& Dickson, B. J. A single class of olfactory neurons mediates behavioural responses to a Drosophila sex pheromone. Nature 446, 542-546 (2007).

36. Letunic, I. \& Bork, P. Interactive Tree Of Life (iTOL) v4: recent updates and new developments. Nucleic Acids Res. 47, W1-W256 (2019).

37. Ebrahim, S. A. et al. Drosophila avoids parasitoids by sensing their semiochemicals via a dedicated olfactory circuit. PLoS Biol. 13, e100213 (2015).

38. Dweck, H. K. et al. Pheromones mediating copulation and attraction in Drosophila. Proc. Natl. Acad. Sci. 112, E2829-E2835 (2015).

39. Lin, C.-C., Prokop-Prigge, K. A., Preti, G. \& Potter, C. J. Food odors trigger Drosophila males to deposit a pheromone that guides aggregation and female oviposition decisions. Elife 4, e08688 (2015).

40. Benton, R., Vannice, K. S., Gomez-Diaz, C. \& Vosshall, L. B. Variant ionotropic glutamate receptors as chemosensory receptors in Drosophila. Cell 136, 149-162 (2009).

41. Silbering, A. F. et al. Complementary function and integrated wiring of the evolutionarily distinct Drosophila olfactory subsystems. J. Neurosci. 31, 13357-13375 (2011).

42. Rytz, R., Croset, V. \& Benton, R. Ionotropic receptors (IRs): chemosensory ionotropic glutamate receptors in Drosophila and beyond. Insect Biochem. Mol. Biol. 43, 888-897 (2013).

43. Jeong, Y. T. et al. An odorant-binding protein required for suppression of sweet taste by bitter chemicals. Neuron 79, 725-737 (2013). 
44. Rihani, K. et al. A conserved odorant binding protein is required for essential amino acid detection in Drosophila. Commun. Biol. 2, 1-10 (2019).

45. Weiss, L. A., Dahanukar, A., Kwon, J. Y., Banerjee, D. \& Carlson, J. R. The molecular and cellular basis of bitter taste in Drosophila. Neuron 69, 258-272 (2011).

46. Menuz, K., Larter, N. K., Park, J. \& Carlson, J. R. An RNA-seq screen of the Drosophila antenna identifies a transporter necessary for ammonia detection. PLoS Genet 10, e1004810 (2014).

47. Faraji, A. \& Unlu, I. The eye of the tiger, the thrill of the fight: effective larval and adult control measures against the Asian tiger mosquito, Aedes albopictus (Diptera: Culicidae), North America. J. Med. Entomol. 53, 1029-1047 (2016).

48. Cork, A. Electroantennographic Responses of New World Screwworm to Components of Swormlure-4 and Related Compounds (IAEAR-6580-F). Report No. IAEA-R--6580-F, 8-14 (International Atomic Energy Agency (IAEA), Vienna, 1992).

49. Green, C., Hall, M., Fergiani, M., Chirico, J. \& Husni, M. Attracting adult New World screwworm, Cochliomyia hominivorax, to odour-baited targets in the field. Med. Vet. Entomol. 7, 59-65 (1993).

50. Torr, S. J. \& Hall, M. J. Odour-baited targets to control New World screwworm, Cochliomyia hominivorax (Diptera: Calliphoridae): a preliminary study. Bull. Entomol. Res. 82, 417-423 (1992).

51. Kaissling, K.-E. Experimental Cell Biology of Taste and Olfaction: Current Techniques and Protocols 361-386 (CRC Press, Boca Raton, 1995)

52. Gomulski, L. M. et al. Transcriptional variation of sensory-related genes in natural populations of Aedes albopictus. BMC Genom. 21, 1-22 (2020).

53. Steinbrecht, R. A. Olfactory Receptors. Atlas of Arthropod Sensory Receptors 155-176 (Springer, New York, 1999).

54. Fleischer, J. \& Krieger, J. Insect pheromone receptors-key elements in sensing intraspecific chemical signals. Front. Cell. Neurosci. 12, 425 (2018).

55. Vosshall, L. B. \& Stocker, R. F. Molecular architecture of smell and taste in Drosophila. Annu. Rev. Neurosci. 30, 505-533 (2007).

56. Fletcher, L., O’Grady, J. Jr., Claborn, H. \& Graham, O. A pheromone from male screw-worm flies. J. Econ. Entomol. 59, 142-143 (1966).

57. Hammack, L. Pheromone-mediated copulatory responses of the screwworm fly, Cochliomyia hominivorax. J. Chem. Ecol. 12, 1623-1631 (1986).

58. Mackley, J. W. \& Broce, A. B. Evidence of a female sex recognition pheromone in the screwworm fly. Environ. Entomol. 10, 406-408 (1981).

59. Carlson, D., Berkebile, D., Skoda, S., Mori, K. \& Mihok, S. Candidate sex pheromones of the New World screwworm Cochliomyia hominivorax. Med. Vet. Entomol. 21, 93-96 (2007).

60. Mori, K., Ohtaki, T., Ohrui, H., Berkebile, D. R. \& Carlson, D. A. Synthesis of the four stereoisomers of 6-acetoxy-19-methylnonacosane, the most potent component of the female sex pheromone of the New World screwworm fly, with special emphasis on partial racemization in the course of catalytic hydrogenation. Eur. J. Org. Chem. 2004, 1089-1096 (2004).

61. Pomonis, J. G., Hammack, L. \& Hakk, H. Identification of compounds in an HPLC fraction from female extracts that elicit mating responses in male screwworm flies, Cochliomyia hominivorax. J. Chem. Ecol. 19, 985-1008 (1993).

62. Paulo, D. F. et al. Specific gene disruption in the major livestock pests Cochliomyia hominivorax and Lucilia cuprina using CRISPR/ Cas9. Genes Genomes Genet. 9, 3045-3055 (2019).

63. Jacob, V. E. Current source density analysis of electroantennogram recordings: a tool for mapping the olfactory response in an insect antenna. Front. Cell. Neurosci. 12, 287 (2018).

64. Olsson, S. B. \& Hansson, B. S. Pheromone Signaling 157-177 (Springer, New York, 2013).

65. Kaissling, K. Handbook of Sensory Physiology (Springer, Berlin, 1971).

66. Kelling, F. J. Chapter 6: Spatial and temporal characteristics of electroantennograms in flies; Thesis: Olfaction in houseflies; $\mathrm{PhD}$ thesis, University of Groningen (2001).

67. Mastrangelo, T. \& Welch, J. B. An overview of the components of AW-IPM campaigns against the New World screwworm. Insects 3, 930-955 (2012).

68. Concha, C. et al. A transgenic male-only strain of the New World screwworm for an improved control program using the sterile insect technique. BMC Biol. 14, 72 (2016).

69. Scheidler, N. H., Liu, C., Hamby, K. A., Zalom, F. G. \& Syed, Z. Volatile codes: correlation of olfactory signals and reception in Drosophila-yeast chemical communication. Sci. Rep. 5, 14059 (2015).

70. Kim, D., Langmead, B. \& Salzberg, S. L. HISAT: a fast spliced aligner with low memory requirements. Nat. Methods 12, 357-360 (2015).

71. Anders, S., Pyl, P. T. \& Huber, W. HTSeq-a Python framework to work with high-throughput sequencing data. Bioinformatics 31, 166-169 (2015).

72. McCarthy, D. J., Chen, Y. \& Smyth, G. K. Differential expression analysis of multifactor RNA-Seq experiments with respect to biological variation. Nucleic Acids Res. 40, 4288-4297 (2012).

73. Robinson, M. D., McCarthy, D. J. \& Smyth, G. K. edgeR: a Bioconductor package for differential expression analysis of digital gene expression data. Bioinformatics 26, 139-140 (2010).

74. Cardoso, G. A., Matiolli, C. C., de Azeredo-Espin, A. M. L. \& Torres, T. T. Selection and validation of reference genes for functional studies in the Calliphoridae family. J. Insect Sci. 14, 1 (2014).

75. Pfaffl, M. W. A new mathematical model for relative quantification in real-time RT-PCR. Nucleic Acids Res. 29, e45-e45 (2001).

76. Galindo, K. \& Smith, D. P. A large family of divergent Drosophila odorant-binding proteins expressed in gustatory and olfactory sensilla. Genetics 159, 1059-1072 (2001).

77. Robertson, H. M., Warr, C. G. \& Carlson, J. R. Molecular evolution of the insect chemoreceptor gene superfamily in Drosophila melanogaster. Proc. Natl. Acad. Sci. 100, 14537-14542 (2003).

78. Thompson, J. D., Gibson, T. J., Plewniak, F., Jeanmougin, F. \& Higgins, D. G. The CLUSTAL_X windows interface: flexible strategies for multiple sequence alignment aided by quality analysis tools. Nucleic Acids Res. 25, 4876-4882 (1997).

79. Stamatakis, A. RAxML version 8: a tool for phylogenetic analysis and post-analysis of large phylogenies. Bioinformatics 30, 13121313 (2014).

\section{Acknowledgements}

We are grateful for technical assistance from Nicolas Mendoza, Domitildo Martinez, Rosaura Sanchez, Hermogenes Gonzalez, Brigido Gonzalez, and Jaime Ceballos at the ARS-COPEG laboratory. Funding is gratefully acknowledged from the cooperative agreements between the USDA-ARS and University of Kentucky (53-30940-013), and from National Institute of Food and Agriculture, US Department of Agriculture (under HATCH Project 2353077000). 


\section{Author contributions}

P.V.H., O.M., A.P.A., A.P.L., W.W., M.J.S. and Z.S. designed research; P.V.H., O.M., A.S. (Panama), A.S. (University of Kentucky)., A.P.L. and Z.S. conducted the research; P.V.H., O.M. and Z.S. analyzed the data; P.V.H., O.M., A.P.A. and Z.S. wrote the paper.

\section{Competing interests}

The authors declare no competing interests.

\section{Additional information}

Supplementary information is available for this paper at https://doi.org/10.1038/s41598-020-77541-w.

Correspondence and requests for materials should be addressed to Z.S.

Reprints and permissions information is available at www.nature.com/reprints.

Publisher's note Springer Nature remains neutral with regard to jurisdictional claims in published maps and institutional affiliations.

(c) (i) Open Access This article is licensed under a Creative Commons Attribution 4.0 International License, which permits use, sharing, adaptation, distribution and reproduction in any medium or format, as long as you give appropriate credit to the original author(s) and the source, provide a link to the Creative Commons licence, and indicate if changes were made. The images or other third party material in this article are included in the article's Creative Commons licence, unless indicated otherwise in a credit line to the material. If material is not included in the article's Creative Commons licence and your intended use is not permitted by statutory regulation or exceeds the permitted use, you will need to obtain permission directly from the copyright holder. To view a copy of this licence, visit http://creativecommons.org/licenses/by/4.0/.

(c) The Author(s) 2020 